\title{
Revascularization of Acute Stent Thrombosis after Carotid Artery Stenting in a Clopidogrel Resistance Patient
}

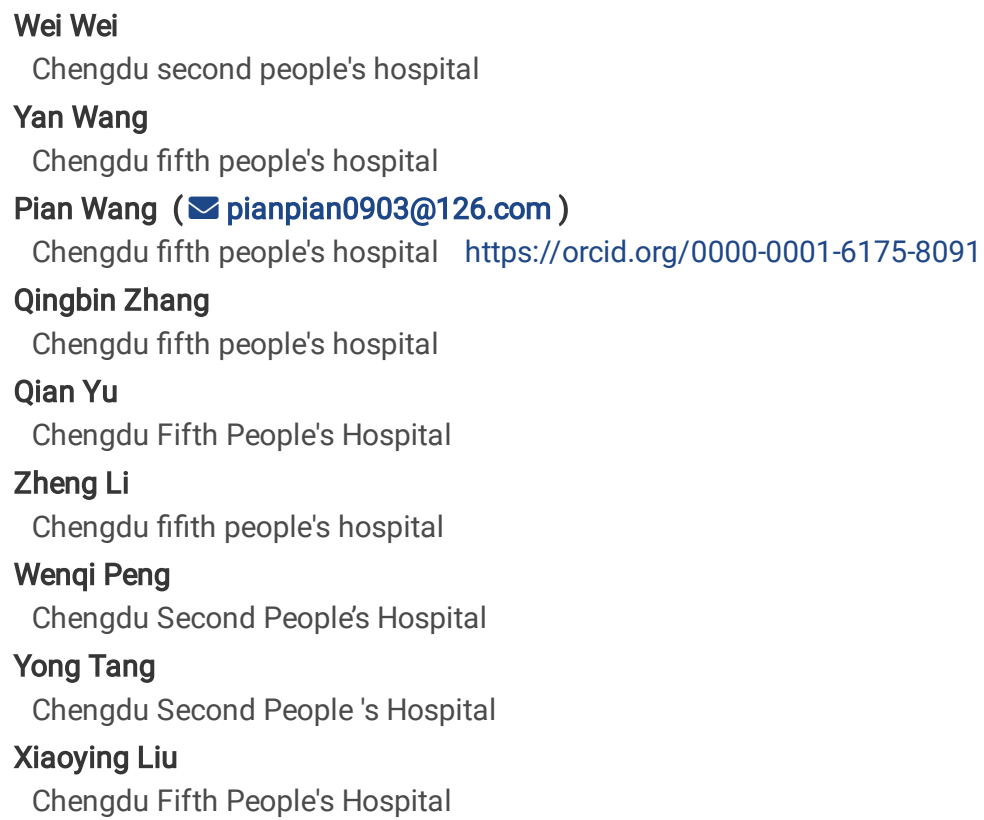

\section{Case report}

Keywords: Acute Stent Thrombosis, Carotid Artery Stenting, revascularization, clopidogrel resistance

Posted Date: October 11th, 2019

DOI: https://doi.org/10.21203/rs.2.1850/v2

License: (c) (1) This work is licensed under a Creative Commons Attribution 4.0 International License. Read Full License 


\section{Abstract}

Carotid artery stenting (CAS) is an alternative strategy to prevent ischemic stroke in patients who are at high risk of surgery compared with carotid endarterectomy (CEA). Acute carotid stent thrombosis (ACST) is an extremely rare but devastating complication after CAS. Theoretically, it occurs within 30 days after CAS. There are several reasons causing ACST, such as inadequate antiplatelet therapy, early discontinuation of antiplatelet therapy, clopidogrel resistance, hypercoagulable state, local vessel dissection, vasospasm, and intimal injury. Although successful recanalization cases have been reported, there is still a lack of experience in the choice of treatment methods and the timing of ACST treatment, especially when the patient has clopidogrel resistance. Here, we report a case with successful revascularization after ACST in a patient with evidenced clopidogrel resistance, which was further confirmed by genetic testing.In this case, both thrombus aspiration and platelet glycoprotein Ilb/llla antagonist (GPIs) were used for recanalization. In addition, we review the literature and discuss appropriate treatment strategies for this devastating and rare event.

\section{Background}

Carotid artery stenting (CAS) is an alternative strategy to prevent primary or secondary ischemic stroke in selected patients compared with carotid endarterectomy (CEA) ${ }^{1}$. It is well-known that acute carotid stent thrombosis (ACST) is an extremely rare event, but it is accompanied by devastating complications ${ }^{2}$. The incidence rate of ACST is $0.5 \%-0.8 \%{ }^{2}$. Although successful recanalization cases have been reported ${ }^{3}{ }^{4}$, there is still a lack of experience in the choice of treatment methods and the timing of ACST treatment, especially when the patient has clopidogrel resistance. Here, we report successful revascularization of ACST in a patient with clopidogrel resistance, and this resistance was further confirmed by genetic testing. We further review the literature and discuss appropriate treatment strategies for this rare event.

\section{Case Report}

The study protocol was approved by the Ethics Committees of the Fifth People's Hospital of Chengdu, Chengdu, and the patient provided written informed consent.

The 69-year-old man was admitted to the Fifth People's Hospital of Chengdu, Chengdu, China for weakness of right limb, right central facial paralysis, and hemianalgesia for 2 days. The weakness of his right limb was aggravated, and barylalia was detected for a half of day. He had a history of hypertension for four years due to poor blood pressure control. After hospitalization, magnetic resonance imaging (MRI) showed multiple internal border zone infarcts in a rosary-like pattern along the left centrum semiovale. A computed tomography (CT) angiography showed severe stenosis at the beginning of the left internal carotid artery (LICA) (Figure 1). His platelet count was $107 \times 10^{9} / \mathrm{L}$, and his coagulation function was normal. After multidisciplinary consultation, including Cardiology, Respiratory Medicine, Neurosurgery, and Anesthesiology, anesthesiologists believed that the risk of general anesthesia was greater due to poor lung function in the patient, resulting in left carotid stenting rather than endarterectomy for secondary prevention. Before the surgery, the patient took aspirin (100 mg) and clopidogrel $(75 \mathrm{mg})$ for 5 days, and atorvastatin $(20 \mathrm{mg})$ was routinely administered for 5 days. The National Institute of Health Stroke Scale (NIHSS) score of the patient was 5.

CAS was performed under local anesthesia. Heparin was administered intravenously at a dose of 5,000 U (100 U/kg) bolus immediately after femoral artery puncture and at a dose of $1,000 \mathrm{U} / \mathrm{h}$ during the procedure. Angiography revealed $90 \%$ stenosis in the left carotid artery according to the North American Symptomatic Carotid Endarterectomy Trial (NASCET) criteria. First, we positioned a distal protection device (spider FX; eV3 Inc., Plymouth, MN, USA), and the stenosis of the left internal carotid artery was then predilated with a $5 \times 30 \mathrm{~mm}$ balloon (Viatrac 14 Plus; Abbott Vascular, Temecula, CA, USA). A $7 \times 40 \mathrm{~mm}$ self-expandable carotid stent (Wallstent; Boston Scientific, Marlborough, MA, USA) was then placed by a 0.014-inch guidewire and an 8-F guide catheter. In addition, the CAS was performed without any complications (Figure 2), and the patient did not show any symptoms during the CAS procedure.

After the surgery, the patient continued to take aspirin $100(\mathrm{mg})$, clopidogrel $(75 \mathrm{mg})$, and atorvastatin $(20 \mathrm{mg})$. One day after surgery, we assayed his platelet count and coagulation function as a routine procedure. His platelet count and coagulation function were both in the normal range. Additionally, the patient did not complain about any discomfort. Five days after surgery, the patient showed lethargy, gaze to the left-side, motor aphasia, and right hemiplegia, and the patient's NIHSS score was 18. After 30 minutes, CT angiography showed acute stent thrombosis of the left internal carotid artery (Figure 3).

We performed thrombus aspiration via right percutaneous transfemoral access under local anesthesia. After the $8 \mathrm{~F}$ guiding catheter (Boston Scientific, Marlborough, MA, USA) was placed, the angiography showed acute thrombosis at the proximal end of the stent without forward blood flow. The Synchro2 Microwire (Stryker, Kalamazoo, MI, USA) with a Rebar 18 microcatheter (ev3 Inc., Irvine, CA, USA) showed thrombosis in the M1 segment. We preferential opening of the MCA blood vessel was conducted through a $4 \times 20 \mathrm{~mm}$ Solitaire FR (ev3 Inc., Irvine, CA, USA) stent with a thrombus approximately 2-3 cm long $\mathbb{F}$ Figure $5 \rrbracket$. A 6F Navien (ev3 Inc., Irvine, CA, USA) was used to enter the stent thrombus for thrombus aspiration, and a thrombus of approximately $4 \mathrm{~cm}$ in length was aspirated as well (Figure 5). After the angiography, the blood flow in the stent was partially restored, but the forward blood flow was still slow. A Spider distal protection device was positioned (ev3 Inc., Irvine, CA, USA), and a $5 \times 30$ $\mathrm{mm}$ balloon (Boston Scientific, Marlborough, MA, USA) was expanded twice by $12 \mathrm{~atm}$. The angiography showed a significant improvement in the anterior blood flow, but there was some thrombus in the stent. Tirofiban $(10 \mathrm{ml})$ was given through a Rebar 18 microcatheter (ev3 Inc., Irvine, CA, 
USA). The angiography then showed complete disappearance of the thrombus and complete recovery of forward blood flow (Figure 5). After surgery, the patient's NIHSS score was 12.

The postoperative CT showed severe cerebral edema and contrast agent leakage without hemorrhage. Two days after thrombus aspiration, the CT angiography showed complete recanalization of the stent, and most of the contrast agents were absorbed. Four days after the second surgery, however, the CT showed a slight hemorrhage in the basal ganglia and cerebral edema around the hemorrhage area. Regarding stent thrombosis, the patient continued to take aspirin, clopidogrel, and atorvastatin. Ten days after the second surgery, we found out that the patient is a CYP $2 \mathrm{C} 19 * 2$ heterozygote. Thus, we gave the patient a triple dose of clopidogrel compared to the preoperative dose ${ }^{5}$. Two weeks after thrombus aspiration, the CT showed hemorrhage absorption. The patient was discharged from the hospital, but he was still taking aspirin, clopidogrel, and atorvastatin. The

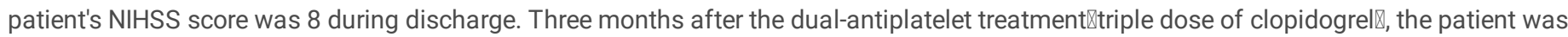
switched to a single-antiplatelet treatment. After 6 months of follow-up, the patient's NIHSS score was 3, and the patient's modified Rankin scale (mRS) score was 1. In addition, there was no stent restenosis in the CT angiography at the 6-month follow-up (Figure 6).

\section{Discussion}

CAS has been confirmed as an alternative strategy to prevent ischemic stroke in patients who are at high-risk of surgery compared with carotid endarterectomy (CEA) ${ }^{1}$. The incidence of in-stent restenosis after CAS was reported to range from $3 \%-16.6 \%$, with the majority of patients being asymptomatic 6 . Acute carotid stent thrombosis (ACST), a major potential complication of CAS, was extremely rare and only occurred within 30 days after the procedure ${ }^{2}$. We reviewed articles related to ACST that were published in English; their details have been listed in Table 1.

Many factors cause ACST, such as inadequate or early discontinuation of antiplatelet therapy, clopidogrel resistance, hypercoagulable state, soft plaque protrusion, local vessel dissection, vasospasm, and intimal injury ${ }^{7-9}$. Also, the stent morphology plays an important role in ACST ${ }^{10}$. Several of these factors (e.g., stent under-expansion, in which the stent does not fully adhere to the blood vessel ${ }^{7}$ or balloon bursts ${ }^{11}$ ), plaque protrusion ${ }^{4}$, and vasospasm can immediately cause ACST, but if promptly treated, can be resolved without any long-term defect to the nervous system. Other factors, such as inadequate or early discontinuation of antiplatelet therapy ${ }^{12-14}$, clopidogrel resistance, and hypercoagulable state ${ }^{8}$, may cause ACST a few days after the CAS procedure. These factors are much more critical and may eventually cause death or severe paralysis ${ }^{12}$.

In our patient, ACST was likely caused by more than one factor. After detection of ACST, we tested for CYP2C19 in our patient and found the presence of CYP2C19*1/*2, a genetic polymorphism conferring clopidogrel resistance. Since we did not detect a hypercoagulable state and antiplatelet therapy was adequate in our patient, we speculate that clopidogrel resistance may be an important contributor to ACST. Furthermore, we did not perform post-stenting balloon dilation, so it is possible that stent under-expansion also contributed to ACST in this patient ${ }^{6}$.

We performed thrombus aspiration immediately after detection of ACST; however, because of slow forward blood flow and the existence of a thrombus in the stent, tirofiban $(10 \mathrm{ml})$ was administered using a Rebar 18 microcatheter. After tirofiban treatment, we performed successful recanalization. As ACST is a rare complication, its treatment is still largely untested. ACST should be treated considering a combination factors such as thrombogenesis, timely action (intraoperative, ideally either just before or after surgery), severity of neurological deficits, and area of infarction. For cases involving severe clinical deterioration, ACST treatment should have relatively positive effects, with the goal of rapid revascularization to avoid serious long-term consequences ${ }^{14}$. Possible solutions for ACST may be multiplex, including the administration of drug ${ }^{4}$

15,16 , thrombolysis ${ }^{11-13,17,18}$, thrombolytic therapy combined with anticoagulation or antiplatelet therapy ${ }^{4}$, and surgical therapy including thromboendarterectomy ${ }^{19}$, mechanical thrombolysis and thrombus aspiration ${ }^{14,20}$, either individually or in combination ${ }^{21}$.

GPIs such as abciximab and tirofiban have successfully been used in the treatment of ACST patients $4,15,16$. In our clopidogrel resistance patient with ACST, the thrombus immediately shrank after tirofiban administration. As tirofiban is a non-peptide tyrosine derivative that mimics the Arg-GlyAsp (RGD) integrin recognition sequence, it has a very short platelet-bound half-life and relatively long plasma half-life. Therefore, its use is advantageous when rapid antiplatelet reversal is required, such as in combination with thrombolysis or thrombus aspiration.

Four days after the second surgery, hemorrhage was detected without any clear cause. Regarding stent thrombosis, dual-antiplatelet therapy was continuously performed囚triple dose of clopidogrel囚. We did not analyze the causes in-depth and did not actively treat cerebral hemorrhage. In other cases, cerebral hemorrhage has not been reported after recanalization. Our patient showed appropriate progress after 6 months of follow-up.

\section{Conclusions}

ACST is an extremely rare event, but fatal complications after CAS and clopidogrel resistance may lead to ACST. The treatment of ACST after CAS must be undertaken urgently and immediately to allow restoration of blood flow and avoid major neurological adverse events. Thrombus aspiration with GPIs would be effective in treatment of ACST. The presented case report only illuminates the available treatment strategies rather than providing general therapeutic recommendations. 


\section{Abbreviations}

Carotid Artery Stenting ,CAS Carotid endarterectomy ,CEA Acute carotid stent thrombosis ,ACSTGlycoprotein Ilb/Illa antagonist ,GPIs Magnetic resonance imaging ,MRI Computed tomography ,CT Left internal carotid artery ,LICA National Institute of Health Stroke Scale ,NIHSS North American Symptomatic Carotid Endarterectomy Trial ,NASCET Middle cerebral artery ,MCA Modified Rankin scale ,Mrs Arg-Gly-Asp ,RGD; Good prognosis: GP; Paresis: P; Death: D.

\section{Declarations}

\section{Consent to publish}

All writers agreed to publish this manuscript. The patient has signed an informed consent form.

\section{Authors Contributions}

Wei Wei and Pian Wang drafted the manuscript for intellectual content, Yan Wang design and conceptualized study, Zheng Li and Qingbin Zhang analyzed the data.

\section{Funding}

No funding was obtained for this study.

\section{Competing Interests}

Non-financial competing interests.

\section{Availability of data and materials}

Access to study data is regulated by Chinese law. Data are available from the Fifth People's Hospital of Chengdu Institutional Data Access/Ethics Committee for researchers who meet the criteria.

\section{Acknowledgments}

None.

\section{References}

1. Massop D, Dave R, Metzger C, et al. Stenting and angioplasty with protection in patients at high-risk for endarterectomy: SAPPHIRE Worldwide Registry first 2,001 patients. Catheterization and cardiovascular interventions : official journal of the Society for Cardiac Angiography \& Interventions 2009;73:129-136.

2. Moulakakis KG, Mylonas SN, Lazaris A, et al. Acute Carotid Stent Thrombosis: A Comprehensive Review. Vascular and endovascular surgery 2016;50:511-521.

3. Arslan S. Reply to the Letter: "Acute Carotid Artery Stent Thrombosis". Cardiovascular and interventional radiology 2018;41:357.

4. Steiner-Boker S, Cejna M, Nasel C, Minar E, Kopp CW. Successful revascularization of acute carotid stent thrombosis by facilitated thrombolysis. AJNR Am J Neuroradiol 2004;25:1411-1413.

5. Mega JL, Hochholzer W, Frelinger AL, 3rd, et al. Dosing clopidogrel based on CYP2C19 genotype and the effect on platelet reactivity in patients with stable cardiovascular disease. JAMA 2011;306:2221-2228.

6. Lal BK, Beach KW, Roubin GS, et al. Restenosis after carotid artery stenting and endarterectomy: a secondary analysis of CREST, a randomised controlled trial. Lancet Neurol 2012;11:755-763.

7. Hu W, Wang L, Wang G. Acute In-Stent Thrombosis after Carotid Angioplasty and Stenting: A Case Report and Literature Review. Interventional neurology 2018;7:265-270.

8. Kanemaru K, Nishiyama Y, Yoshioka H, et al. In-stent thrombosis after carotid artery stenting despite sufficient antiplatelet therapy in a bladder cancer patient. Journal of stroke and cerebrovascular diseases : the official journal of National Stroke Association 2013;22:1196-1200.

9. Okazaki T, Satomi J, Satoh K, Hirasawa M, Nagahiro S. Rescue revascularization therapy with a stent-in-stent technique for acute intracranial internal carotid artery occlusion. Neurologia medico-chirurgica 2005;45:253-258.

10. Musialek P, Kolvenbach R, Schofer J. Letter by Musialek et al Regarding Article, "Acute Occlusions of Dual-Layer Carotid Stents After Endovascular Emergency Treatment of Tandem Lesions". Stroke 2017;48:e364.

11. lancu A, Grosz C, Lazar A. Acute carotid stent thrombosis: review of the literature and long-term follow-up. Cardiovasc Revasc Med 2010;11:110-113 
12. Chaturvedi S, Sohrab S, Tselis A. Carotid stent thrombosis: report of 2 fatal cases. Stroke 2001;32:2700-2702.

13. Hamann GF, Liebetrau M, Pfefferkorn T, Heiss MM. Successful systemic thrombolysis for carotid stent thrombosis. Eur Neurol 2002;48:37-39.

14. Masuo O, Terada T, Matsuda Y, et al. Successful recanalization by in-stent percutaneous transluminal angioplasty with distal protection for acute carotid stent thrombosis. Neurologia medico-chirurgica 2006;46:495-499.

15. Tong FC, Cloft HJ, Joseph GJ, Samuels OB, Dion JE. Abciximab rescue in acute carotid stent thrombosis. AJNR Am J Neuroradiol 2000;21:1750-1752.

16. Seo KD, Lee KO, Kim DJ, Lee KY. Rescue use of tirofiban for acute carotid in-stent thrombosis. Yonsei Med J 2008;49:163-166.

17. Moulakakis KG, Kakisis J, Tsivgoulis G, et al. Acute Early Carotid Stent Thrombosis: A Case Series. Ann Vasc Surg 2017;45:69-78.

18. Dhall A, Malani SK, Chadha DS. Thrombosuction for procedural acute thrombosis during high-risk carotid angioplasty-a case report. J Invasive Cardiol 2010;22:E144-146.

19. Setacci C, de Donato G, Setacci F, et al. Surgical management of acute carotid thrombosis after carotid stenting: a report of three cases. J Vasc Surg 2005;42:993-996.

20. Kim YW, Kang DH, Hwang JH, Park J, Hwang YH, Kim YS. Rescue strategy for acute carotid stent thrombosis during carotid stenting with distal filter protection using forced arterial suction thrombectomy with a reperfusion catheter of the Penumbra System: a technical note. Acta Neurochir (Wien) 2013;155:1583-1588.

21. Moulakakis KG, Lazaris AM. Emergent Carotid Stent Removal after Carotid Stent Thrombosis. Ann Vasc Surg 2018;46:401-406.

22. Owens EL, Kumins NH, Bergan JJ, Sparks SR. Surgical management of acute complications and critical restenosis following carotid artery stenting. Annals of vascular surgery 2002;16:168-175.

23. Bush RL, Bhama JK, Lin PH, Lumsden AB. Transient ischemic attack due to early carotid stent thrombosis: successful rescue with rheolytic thrombectomy and systemic abciximab. Journal of endovascular therapy : an official journal of the International Society of Endovascular Specialists 2003;10:870-874.

24. Buhk JH, Wellmer A, Knauth M. Late in-stent thrombosis following carotid angioplasty and stenting. Neurology 2006;66:1594-1596.

25. Choi HJ, Kim ST, Jeong YG, Jeong HW. Superficial temporal artery-middle cerebral artery anastomosis for internal carotid artery occlusion by subacute in-stent thrombosis after carotid artery stenting. Journal of Korean Neurosurgical Society 2012;52:551-554.

26. Markatis F, Petrosyan A, Abdulamit T, Bergeron P. Acute carotid stent thrombosis: a case of surgical revascularization and review of treatment options. Vascular 2012;20:217-220.

27. Munich S, Moftakhar R, Lopes D. Recanalization of acute carotid stent occlusion using Penumbra 4Max aspiration catheter: technical report and review of rescue strategies for acute carotid stent occlusion. Journal of neurointerventional surgery 2014;6:e42.

28. Koklu E, Arslan S, Yuksel IO, Bayar N, Koc P. Acute Carotid Artery Stent Thrombosis Due to Dual Antiplatelet Resistance. Cardiovascular and interventional radiology 2015;38:1011-1014.

\section{Tables}

Table 1. Acute stent thrombosis after carotid artery stenting 


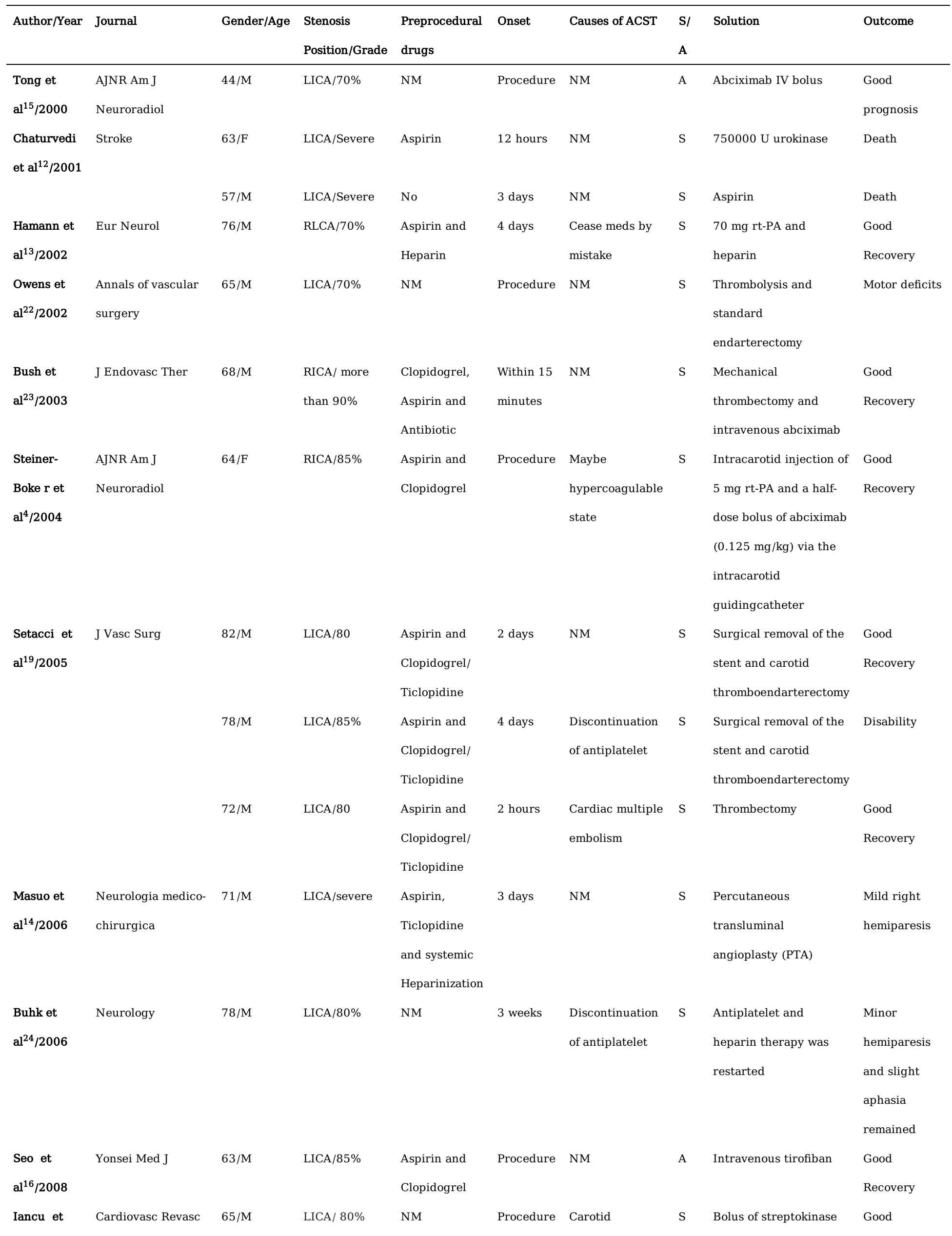




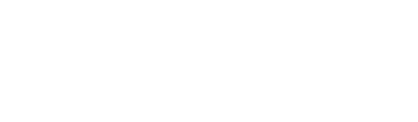

70/M

RICA/80\%

NM

following stent

postdilation

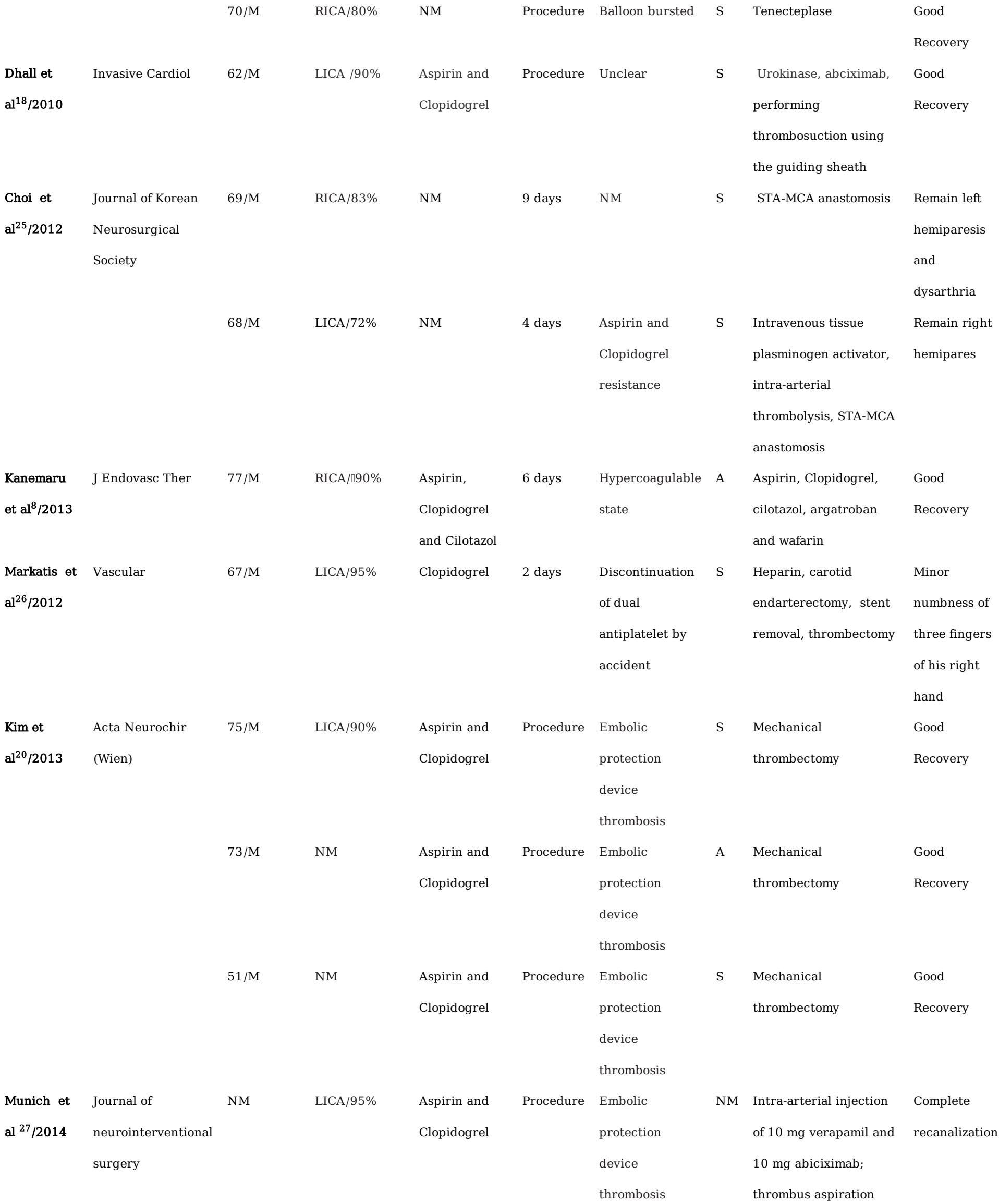




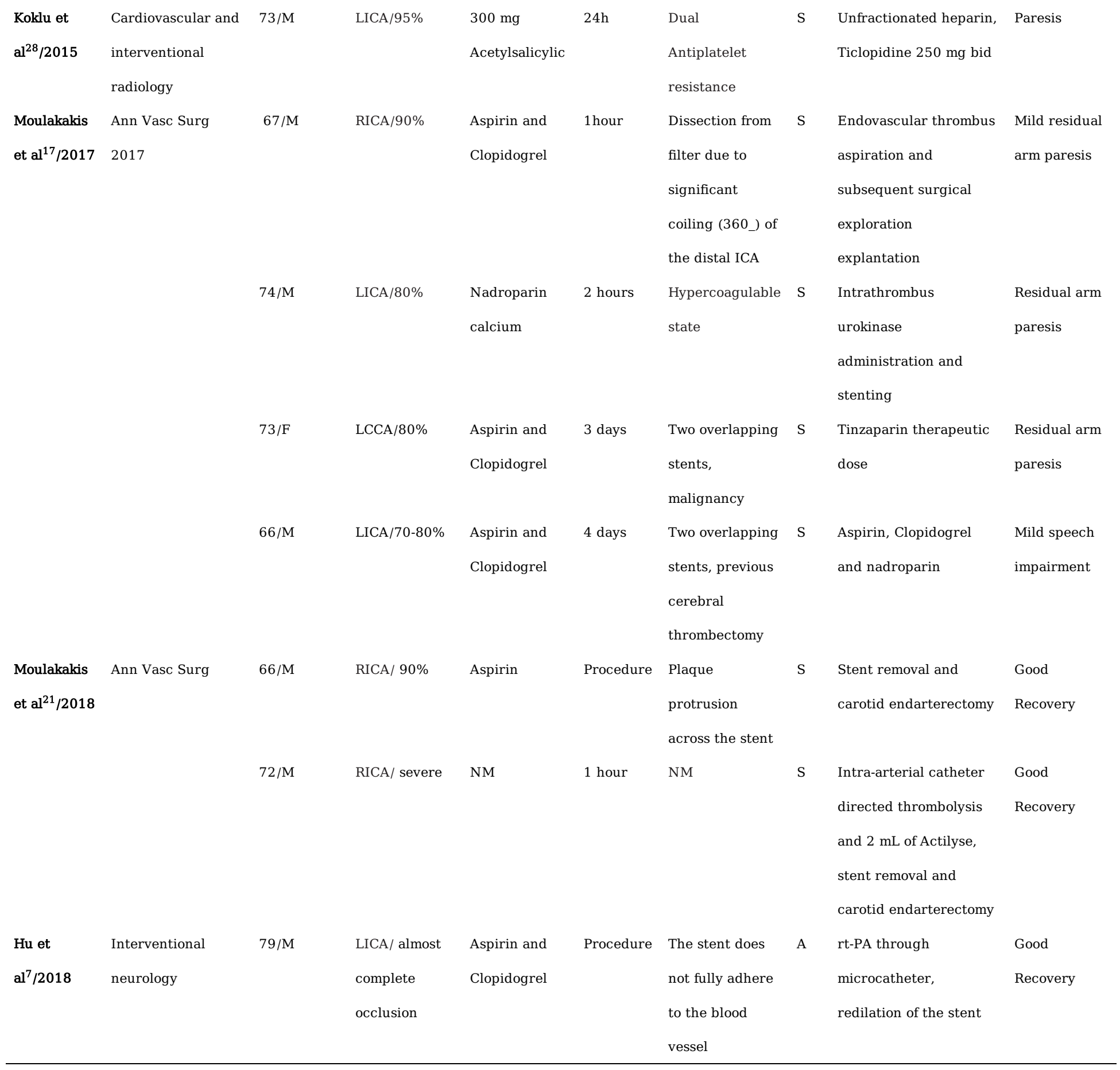

Not Mentioned: NM; Symptomatic: S; Asymptomatic: A

\section{Figures}




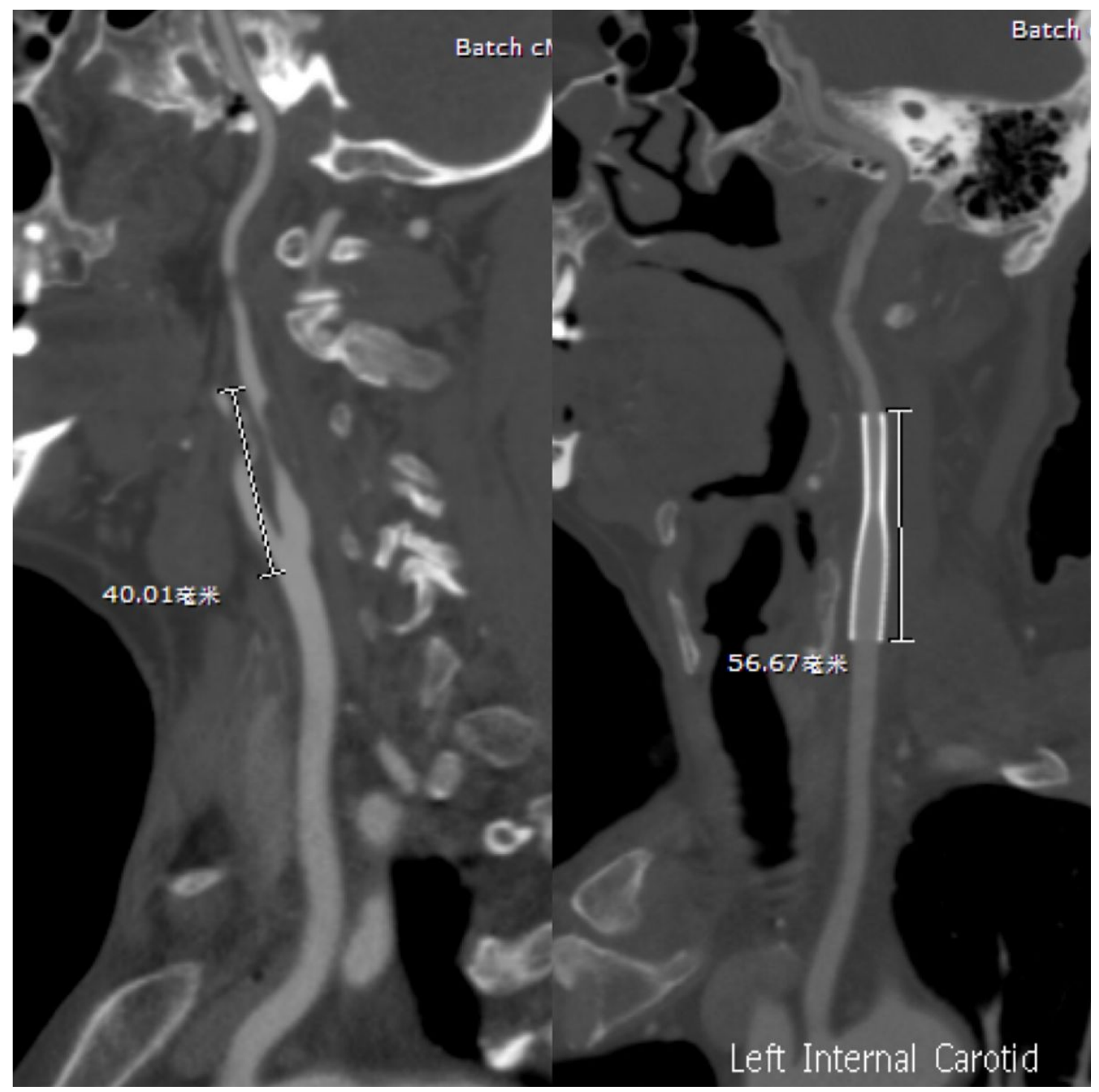

\section{Figure 1}

(A) Magnetic resonance imaging (MRI) showed multiple internal border zone infarcts in a rosary-like pattern along the left centrum semiovale; (B, C) The computed tomography (CT) angiography showed severe stenosis at the beginning of the left internal carotid artery (LICA); (D) The doppler ultrasonography showed the formation of an atherosclerotic plaque in the LICA and accelerated blood flow velocity in the lumen. 

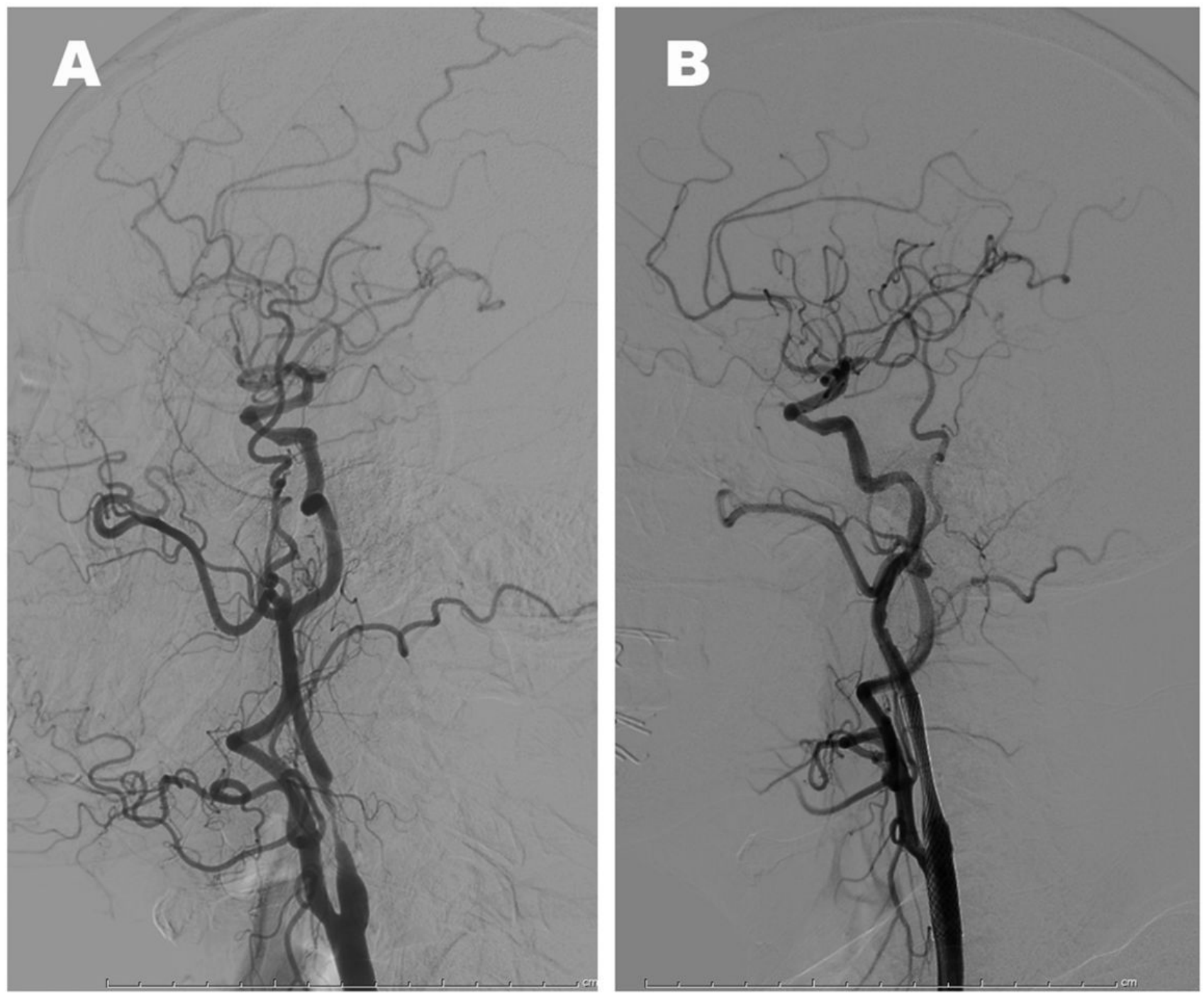

\section{Figure 2}

(A) Angiography revealed $90 \%$ stenosis in left carotid artery according to The North American Symptomatic Carotid Endarterectomy Trial criteria;

(B) CAS was performed without any complication, and the stenosis of LICA was fully solved. Figure 35 days after surgery, CT angiography showed acute stent thrombosis of the left internal carotid artery. 


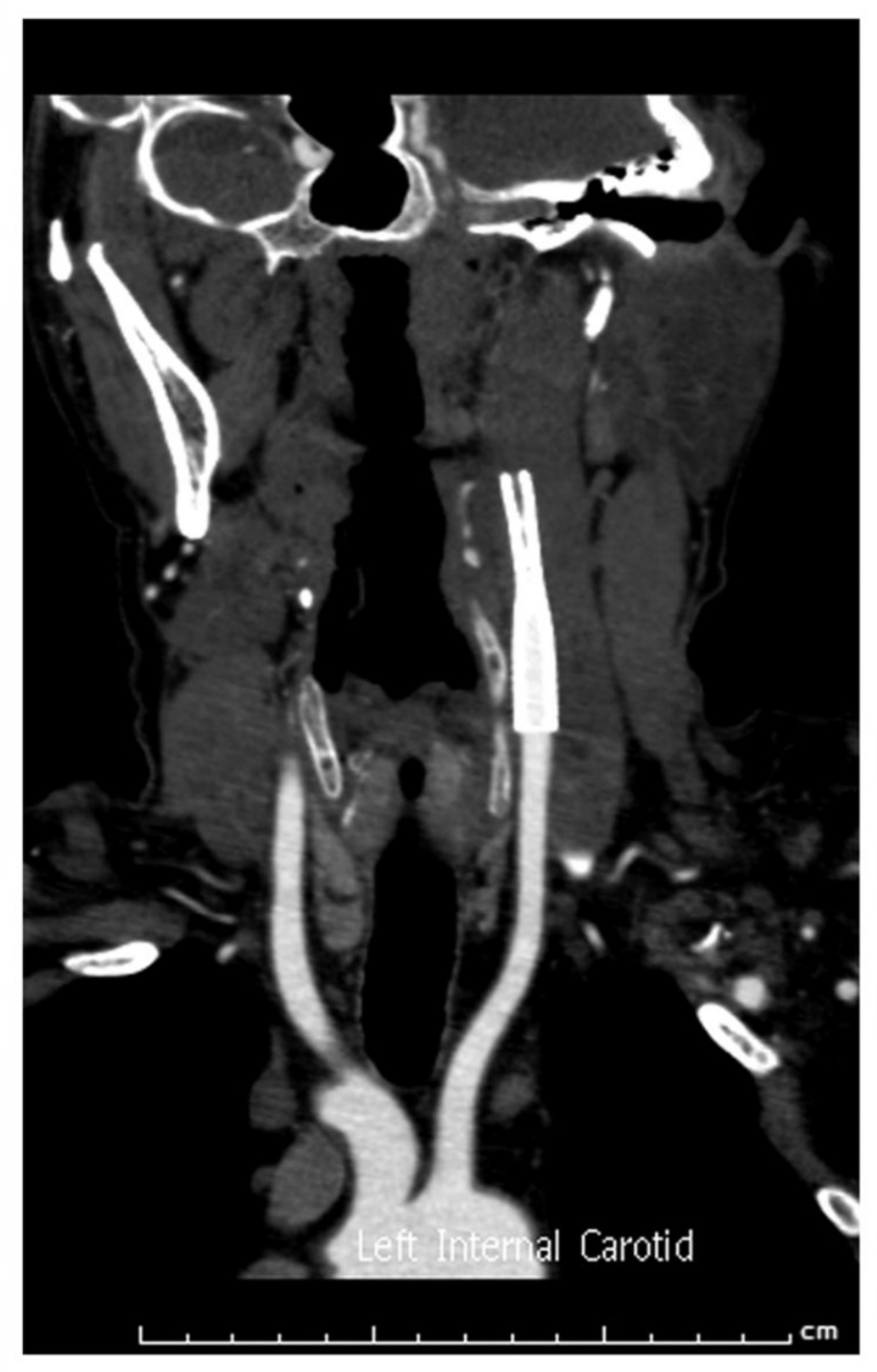

Figure 3

5 days after surgery, CT angiography showed acute stent thrombosis of the left internal carotid artery. 

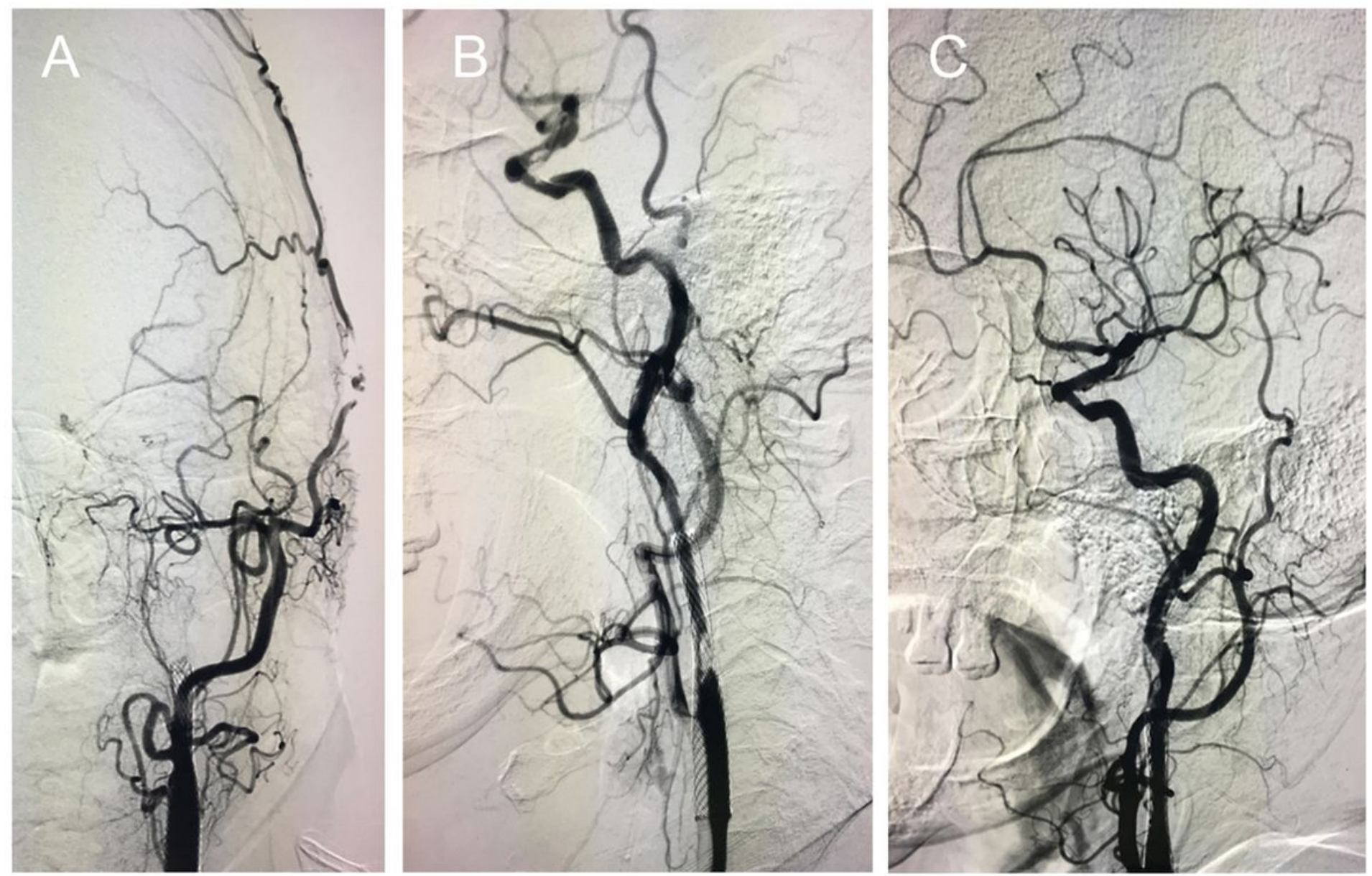

\section{Figure 4}

The thrombus aspiration was carried out for the patient with acute carotid stent thrombosis. (A) Angiography confirmed acute stent thrombosis of the left internal carotid artery, however, there was no blood flue at the distal end of the stent; (B) After thrombus aspiration, the blood flow in the stent was partially restored, while the forward blood flow was still very slow and there were some thrombus in the stent; (C) The acute carotid thrombosis was fully solved after balloon expansion, and Tirofiban (10 ml) was given through Rebar18 microcatheter. 


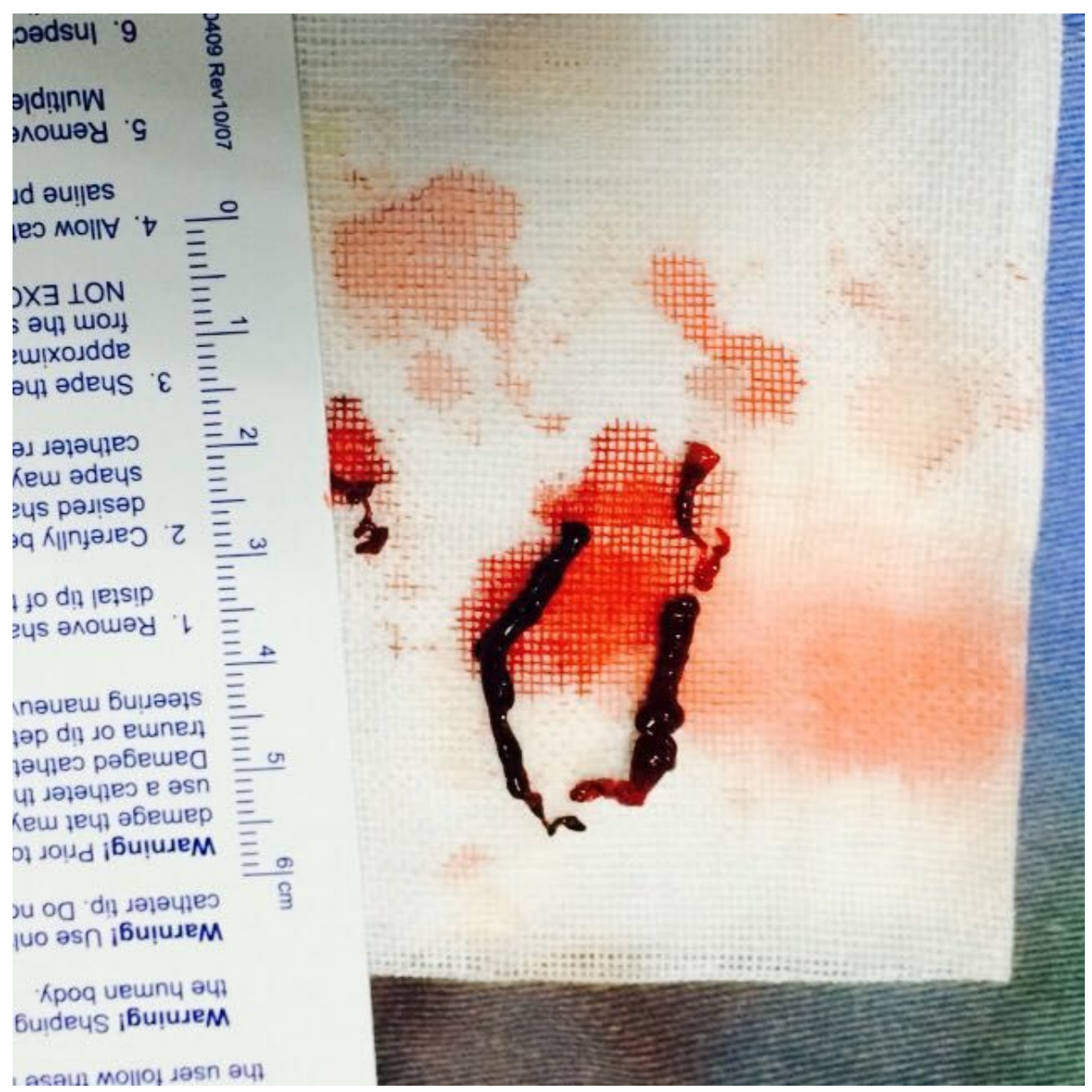

\section{Figure 5}

Extracted thrombus in this ACST patient. 

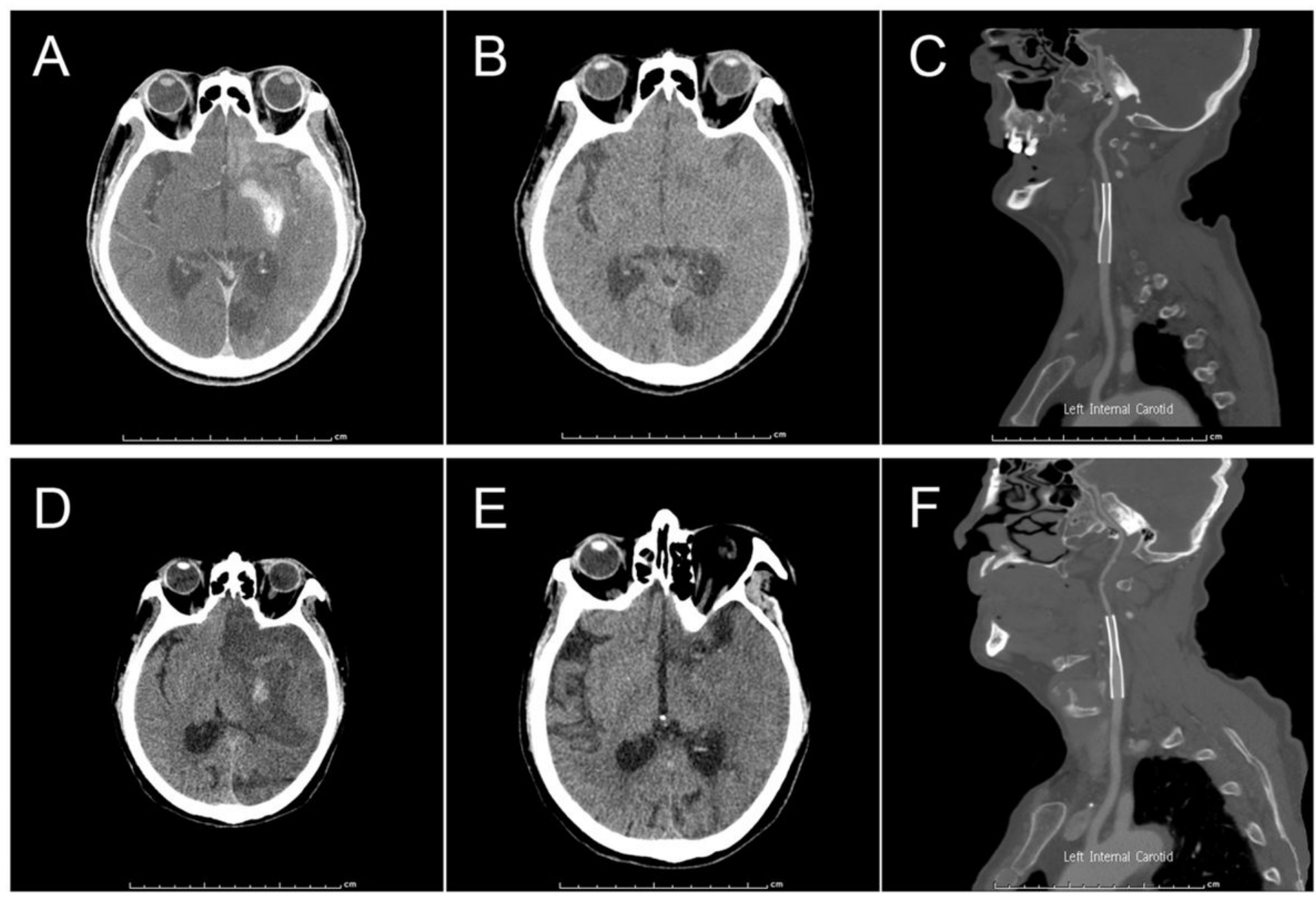

Figure 6

A-F shows the radiographic changes in the patient after thrombus aspiration. (A) The postoperative CT showed contrast agent leakage; (B,C) Two days after thrombus aspiration, the CT angiography showed complete recanalization of the stent, and most of the contrast agents were absorbed; (D) Four days after the second surgery, the CT showed a slight hemorrhage in basal ganglia and cerebral edema around the hemorrhage area; (E) Two weeks after thrombus aspiration, the CT showed hemorrhage absorption; (F) There was no stent restenosis in the CT angiography after 6 months of follow-up. 